\title{
Disulfide-Based Poly(amido amine)s for siRNA Delivery: Effects of Structure on siRNA Complexation, Cellular Uptake, Gene Silencing and Toxicity
}

\author{
Pieter Vader • Leonardus J. van der Aa • Johan F. J. Engbersen • Gert Storm • Raymond M. Schiffelers
}

Received: 6 October 2010 / Accepted: 1 December 2010 / Published online: 23 December 2010

(C) The Author(s) 2010. This article is published with open access at Springerlink.com

\begin{abstract}
Purpose RNA interference (RNAi) is a process by which small interfering RNAs (siRNA) induce sequence-specific gene silencing. Therefore, siRNA is an emerging promise as a novel therapeutic. In order to realize the high expectations for therapeutic applications, efficient delivery systems for siRNA are necessary.

Methods In this study, a new series of biodegradable poly (amido amine)s with disulfide linkages in the backbone was synthesized out of $\mathrm{N}, \mathrm{N}^{\prime}$-cystaminebisacrylamide (CBA), 4amino-I-butanol (ABOL) and ethylene diamine (EDA). Effects of different percentages of butanolic side chains and protonatable fragments in the main chain on siRNA complexation, cellular uptake, gene silencing and toxicity were investigated.
\end{abstract}

Results Incorporation of EDA in the polymer resulted in increased siRNA condensation. Efficient siRNA condensation

Electronic Supplementary Material The online version of this article (doi: I 0. I007/s I | 095-0 I0-0344-y) contains supplementary material,

which is available to authorized users.

P. Vader $\cdot$ G. Storm $\cdot$ R. M. Schiffelers

Department of Pharmaceutics Z-513

Utrecht Institute for Pharmaceutical Sciences (UIPS)

Utrecht University

P.O. Box 80082, 3508 TB Utrecht, The Netherlands

L. J. van $\operatorname{der} \mathrm{Aa} \cdot$ J. F. J. Engbersen

Depts. of Biomedical Chemistry \& Polymer Chemistry \& Biomaterials

MIRA Institute for Biomedical Technology \& Technical Medicine

Faculty of Science \& Technology

University of Twente

P.O. Box 217, 7500 AE Enschede, The Netherlands

P. Vader $(\square)$

Faculty of Science, Department of Pharmaceutics Z-513

Utrecht Institute for Pharmaceutical Sciences (UIPS)

Utrecht University

Sorbonnelaan 16

3584 CA Utrecht, The Netherlands

e-mail: P.Vader@uu.nl was shown to be necessary for cellular uptake; however, excess of polymer decreased siRNA uptake for polymers with high amounts of EDA. Silencing efficiency did not correlate with uptake, since silencing increased with increasing $\mathrm{w} / \mathrm{w}$ ratio for all polymers. More than $80 \%$ knockdown was found for polyplexes formed with polymers containing $25 \%$ or $50 \%$ EDA, which was combined with low cytotoxicity.

Conclusions Poly(amido amine)s with minor fractions of protonatable fragments in the main chain are promising carriers for delivery of siRNA.

KEY WORDS delivery - disulfide bonds · poly(amido amine)s . quantitative uptake $\cdot$ siRNA

\section{INTRODUCTION}

RNA interference (RNAi) is an evolutionary conserved process for post-transcriptional silencing $(1,2)$. Introduction of short interfering RNA (siRNA) molecules into cells can effectuate RNAi (3). The technique is currently widely used as a tool in functional genomics, but it also holds great promise as a therapeutic strategy, by suppressing the expression of diseaserelated genes $(4,5)$. However, since siRNA molecules are relatively large and highly negatively charged, they are not readily taken up by cells. Therefore, the development of efficient and safe delivery systems that deliver siRNA to its site of action, i.e. the cytoplasm, is essential for therapeutic activity. These systems should fulfill several requirements, including the ability to protect siRNA during the extracellular delivery stage, enhance cellular association and uptake, trigger endosomal escape and release siRNA in the cytoplasm. For clinical use, they should furthermore be safe and biodegradable. Current methods for siRNA delivery include viral vectors, peptides (6), cationic lipids (7), liposomes (8) and 
cationic polymers such as poly(ethylenimine) (PEI) (9) and poly(L-lysine) (PLL) (10). Although these approaches have all shown specific advantages and disadvantages, the attractiveness of polymers lies in the possibility to be specifically tailored for different applications. At the same time, the major drawback of the currently used polymers is their high toxicity, which is most likely caused by their poor biodegradability.

Recently, a new class of biodegradable cationic polymers based on poly(amido amine)s with disulfide linkages in the backbone (SS-PAA polymers) has been developed (11). These polymers self assemble with plasmid DNA into nanosized polyplexes and display efficient gene transfer properties. Due to the difference in redox potential between the oxidizing extracellular space and the reducing intracellular space, the disulfide bonds are stable outside the cell, but are rapidly cleaved in the cytoplasm. Introduction of disulfide linkages in the polymer chain has already been shown to result in increased transfection efficiency, due to an increased release of DNA from the complexes and decreased toxicity for this class of polymers (12). From the SS-PAAs tested by Lin et al., the copolymer of N,N'cystaminebisacrylamide (CBA) and 4-amino-1-butanol (ABOL) showed the best balance between DNA transfection efficiency and toxicity (12). The origin of the positive effect of the butanolic side chains of this polymer on transfection efficiency still has to be elucidated; however, it has also been found for a similar class of polymers (13).

Although delivery of DNA and siRNA faces the same challenges, their molecular topography is different, which highlights different requirements to their respective delivery systems (14). In this study, CBA was copolymerized besides ABOL with 1,2-diaminoethane (EDA) as the amine monomer to introduce more positive charges in the polymer (Fig. 1). This is expected to increase the electrostatic interactions between polymer and siRNA, resulting in improved complexation. Copolymers containing different percentages of butanolic side chains and aminoethyl frag- ments in the main chain were synthesized and evaluated for their potential use for siRNA delivery by comparing their siRNA condensation properties, cellular uptake, gene silencing efficiency and toxicity.

\section{MATERIALS AND METHODS}

\section{siRNAs}

siRNAs were chemically synthesized and supplied by Eurogentec (Maastricht, The Netherlands). The sequence of epidermal growth factor receptor (EGFR) siRNA was 5'GUU-UGC-CAA-GGC-ACG-AGU-AdTdT-3'; 3'dTdTC-AAA-CGG-UUC-CGU-GCU-CAU-5'. For uptake studies, the $5^{\prime}$-end of the sense strand was modified with Alexa 488 dye. Negative control siRNA (Eurogentec) was used for gel retardation assays, size and zeta potential measurements and cell viability experiments.

\section{Polymer Synthesis and Characterization}

The poly(amidoamine) copolymers were synthesized by Michael addition polymerization reactions as previously described $(12,15)$. However, the amine monomers 4-amino1-butanol (ABOL) and 1,2-diaminoethane (EDA) were added sequentially to the reaction mixture. This is necessary, since EDA contains two primary amino groups and, therefore, in principle, can react four times with acrylamide moieties in the Michael addition, which results in branching and formation of networks. By first allowing all ABOL monomer to react with the acrylamide groups to form linear prepolymer chains with acrylamide terminal groups and subsequently adding an equimolar amount of EDA, branching is prevented as much as possible, since the primary amines in EDA have a higher reactivity than the newly formed secondary amines in the polymer chain.<smiles>C=CC(=O)NCCSSCCNC(=O)C=C</smiles>

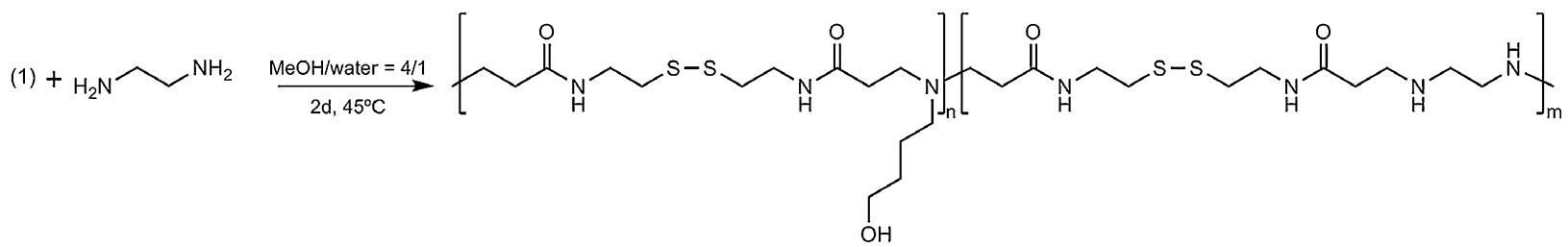

Fig. I Synthesis of poly(CBA-ABOL/EDA) copolymers by prepolymerization of $C B A$ with $A B O L$ and subsequently with EDA monomers. 
Therefore, $\mathcal{N}, \mathcal{N}^{\prime}$-cystaminebisacrylamide $(\mathrm{CBA})$ and $\mathrm{ABOL}$ were first prepolymerized in methanol / water $(4 / 1)$ at $45^{\circ}$ C (Fig. 1). After six days of polymerization, EDA was added, and after two days, the polymerization was terminated by addition of an excess of EDA. The product was purified by ultrafiltration (MWCO 1000, pH 5), filtered through a $0.45 \mu \mathrm{m}$ filter and recovered by lyophilization, and analyzed by ${ }^{1} \mathrm{H}$ NMR and GPC.

\section{Polyplex Formation}

For polyplexes at different polymer/siRNA (w/w) ratios, siRNA and the appropriate amount of polymer solution were each diluted in HBG (Hepes-Glucose buffer: $20 \mathrm{mM}$ Hepes, $\mathrm{pH} 7.4,5 \%$ glucose). Next, the polymer solution was added to the siRNA solution in an Eppendorf tube, at a volume ratio of polymer solution to siRNA solution of 4:1. The mixture was vortexed for $5 \mathrm{~s}$ and incubated for $30 \mathrm{~min}$ at room temperature before use. Lipoplexes with Lipofectamine2000 (Invitrogen, Breda, The Netherlands), used as a control, were prepared at the optimal Lipofectamine2000:siRNA ratio, according to the manufacturer's protocol.

\section{Gel Retardation Assay}

Polyplexes, containing 125 pmol siRNA, were prepared in $50 \mu \mathrm{l} \mathrm{HBG}$. Polyplexes were incubated for $1 \mathrm{~h}$ at $37^{\circ} \mathrm{C}$ in the presence or absence of $5 \mathrm{mM}$ glutathione. Next, $4 \mu \mathrm{l}$ $6 \times$ loading dye (Fermentas) was added to $20 \mu \mathrm{l}$ of polyplex solution, and samples were loaded on a $4 \%$ agarose gel containing $0.5 \mu \mathrm{g} / \mathrm{ml}$ ethidium bromide. Electrophoresis was performed at $90 \mathrm{~V}$ for $30 \mathrm{~min}$, after which pictures of the gel were made under UV-illumination.

\section{Size and $\zeta$-potential Measurements}

Polyplexes, containing 200 pmol siRNA, were prepared in $500 \mu \mathrm{l} \mathrm{HBG}$. Hydrodynamic diameters were determined using dynamic light scattering on an ALV CGS-3 system (Malvern Instruments Ltd., Worcestershire, United Kingdom). For $\zeta$-potential measurements, polyplexes were 1:1 diluted in HBG and measured by laser Doppler electrophoresis using a Zetasizer Nano-Z (Malvern Instruments Ltd., Worcestershire, United Kingdom).

\section{Cell Culture}

The human head and neck squamous cell carcinoma (HNSCG) cell line UM-SCG-14 C, abbreviated as 14 C, was kindly provided by Prof. Dr. G.A.M.S. van Dongen (Department of Otolaryngology, Head and Neck Surgery, VU Medical Center, Amsterdam, The Netherlands). Cells were cultivated at $37^{\circ} \mathrm{C}$ and $5 \% \mathrm{CO}_{2}$ in Dulbecco's Modified Eagle's medium
(DMEM) containing $3.7 \mathrm{~g} /$ liter sodium bicarbonate, $4.5 \mathrm{~g} /$ liter L-glucose and $2 \mathrm{mM}$ L-glutamine, supplemented with $5 \%(v / v)$ fetal bovine serum, $100 \mathrm{IU} / \mathrm{ml}$ penicillin, $100 \mu \mathrm{g} / \mathrm{ml}$ streptomycin and $0.25 \mu \mathrm{g} / \mathrm{ml}$ amphotericin B.

\section{Cellular Uptake Measurements}

\section{Qualitative Uptake}

Fourteen C cells $\left(8 \times 10^{3}\right.$ per well $)$ were seeded in 12-well plates on coverslips, $48 \mathrm{~h}$ before transfection. Medium was replaced with serum-free medium, and cells were treated with $20 \mu$ l of different polyplexes, at a final concentration of $66 \mathrm{nM}$ fluorescently labeled siRNA. After $4 \mathrm{~h}$, cells were washed twice with phosphate-buffered saline (PBS) and fixed with $4 \%$ paraformaldehyde in PBS at room temperature for $30 \mathrm{~min}$. After fixation, cells were washed twice with PBS, mounted on glass cover slides using FluorSave (Calbiochem, San Diego, CA, USA) and allowed to air dry overnight. Cells were then imaged by a Nikon TE2000-U fluorescent microscope, equipped with a GFP-B filter (Nikon Benelux, Brussels, Belgium).

\section{Quantitative Uptake}

Quantitative uptake of siRNA was determined as previously described, with minor modifications (16). Fourteen $\mathrm{C}$ cells $\left(1.6 \times 10^{5}\right.$ cells per well $)$ were seeded in 6 -well plates, $24 \mathrm{~h}$ before transfection. Medium was replaced with serum-free medium, and cells were treated with $400 \mu \mathrm{l}$ of different polyplexes, at a final concentration of $66 \mathrm{nM}$ fluorescently labeled siRNA. After $4 \mathrm{~h}$, cells were put on ice and washed with PBS, $1 \mathrm{M} \mathrm{NaCl}$ and again PBS to remove all noninternalized complexes (Supplementary Fig. 1). Four-hundred $\mu 1$ lysis buffer ( $2 \%$ SDS, $1 \%$ Triton X-100 in PBS) was added to solubilize cells and dissociate polyplexes. Cells were lysed for $1 \mathrm{~h}$ on ice, after which the lysate was centrifuged (15 min, $14,000 \mathrm{~g}, 4^{\circ} \mathrm{C}$ ) to remove cell debris. Two-hundred $\mu \mathrm{l}$ of the supernatant was transferred to a black 96 -well plate to measure fluorescence on a Mithras LB 940 (Berthold Technologies, Bad Wildbad, Germany). A calibration curve of fluorescent siRNA in lysis buffer was used to calculate the amount of internalized siRNA. Aliquots $(25 \mu \mathrm{L})$ of the supernatants were used to determine the cellular protein content using the Micro BCA ${ }^{\mathrm{TM}}$ protein assay (Pierce, Rockford, USA), according to the instructions of the supplier. The amount of internalized siRNA was normalized to the amount of protein, and uptake was calculated as percentage of total siRNA input.

\section{Gene Silencing Experiments}

Silencing experiments were performed as previously described (17). Fourteen $\mathrm{C}$ cells $\left(4 \times 10^{4}\right.$ cells per well) were 
seeded in 24-well plates, $24 \mathrm{~h}$ before transfection. Medium was replaced with serum-free medium, and cells were treated with $100 \mu \mathrm{l}$ of different polyplexes, at a final concentration of $66 \mathrm{nM}$ anti-EGFR siRNA. After $4 \mathrm{~h}$, medium was replaced with complete medium, and cells were incubated for another $48 \mathrm{~h}$. Cells were trypsinized, washed with cold PBA buffer $(0.3 \%$ BSA, $0.03 \%$ sodium azide in PBS) and incubated with a specific FITC-labeled anti-EGFR monoclonal antibody (Santa Cruz Biotechnology Inc., Santa Cruz, CA, USA) in $150 \mu$ l PBA buffer for $30 \mathrm{~min}$ at $4^{\circ} \mathrm{C}$. After that, cells were washed twice with cold PBA buffer and resuspended in $300 \mu \mathrm{l}$ PBA buffer. Measurements were taken on a FACSCalibur (Becton \& Dickinson, Mountain View, CA, USA) and analyzed using WinMDI 2.9 (C1993-2000 Joseph Trotter). In a density plot representing forward scatter and sideward scatter, whole cells were gated out, and at least 5,000 cells were counted for each sample. The mean fluorescent intensity (MFI) served as a measure of the amount of EGFR present on the cells.

\section{Cell Proliferation Assay}

Fourteen C cells $\left(8 \times 10^{3}\right.$ cells per well $)$ were seeded in 96 well plates, $24 \mathrm{~h}$ before transfection. Medium was replaced with serum-free medium, and cells were treated with $20 \mu \mathrm{l}$ of different polyplexes, at a final concentration of $66 \mathrm{nM}$ siRNA. After $4 \mathrm{~h}$, medium was replaced with complete medium, and cells were incubated for $24 \mathrm{~h}$. BrdU reagent was added to a final concentration of $10 \mu \mathrm{M}$, and cells were cultured for another $24 \mathrm{~h}$. Colorimetric BrdU cell proliferation assay (Roche Applied Science, Penzberg, Germany) was performed according to the supplier's instructions.

\section{Statistical Analysis}

Results were analyzed using ANOVA with Bonferroni posttests to assess statistical significance.

\section{RESULTS AND DISCUSSION}

In this study, a new series of poly(amido amine) copolymers was explored, based on the copolymer of N,N'-cystamine- bisacrylamide (CBA) and 4-amino-1-butanol (ABOL), which has recently been shown to efficiently deliver plasmid DNA to COS-7 cells (12). This polymer was tailored for siRNA delivery by copolymerizing 1,2-diaminoethane (EDA) beside ABOL to increase the density of amine groups in the main chain of the polymer. Since previous studies have already shown that small changes in the structure of the polymer can affect the delivery efficiency $(11,18)$, copolymers containing different percentages of $\mathrm{ABOL}$ and EDA fragments were synthesized. The characteristics of the final polymers, poly(CBA-ABOL/EDA), are shown in Table I. The compositions of the polymers were in accordance with the aimed composition, molecular weights ranged between 1.6 and $11.7 \mathrm{kDa}$ and polydispersity indices were comparable for all polymers.

\section{Polyplex Formation}

Formation of polyplexes was confirmed by gel retardation. When siRNA is efficiently bound to its carrier and subsequently shielded from the environment, migration into the gel is completely retarded. Polyplexes were formed at $\mathrm{w} / \mathrm{w}$ ratios 0 (naked siRNA) to 48. Results are shown in Fig. 2, left panel. Poly(CBA-ABOL) could not efficiently encapsulate siRNA. Only at the highest w/w ratio tested, siRNA was completely bound to its carrier (Fig. 2A, left panel). As hypothesized, addition of EDA to the final copolymer resulted in increased complexation of siRNA at lower w/w ratios. Complete retardation was achieved at w/w ratios 12 and higher for polymers with $25 \% \mathrm{EDA}$ and 6 and higher for polymers with $50 \%$ and $75 \%$ EDA, respectively (Fig. 2B, C, D, left panel). Poly(CBA-EDA) showed efficient complexation at w/w ratio 3 and higher (Fig. 2E, left panel). This increased condensation ability with increasing percentage of EDA in the main chain of the polymer can be explained by the increased charge density of the polymer, since, after incorporation of EDA, increased complexation of siRNA was observed at lower w/w ratios.

Although for cellular uptake and protection against nucleases, siRNA has to be complexed, for efficient gene silencing, siRNA should be completely released from the complex inside the cell $(15,19)$. One of the microenvironmental features which has been exploited for
Table I Characteristics of Synthesized CBA-ABOL/EDA Copolymers

${ }^{a}$ Stoichiometric ratio

${ }^{b}$ Determined by ${ }^{1} \mathrm{H}$ NMR

${ }^{c}$ After ultrafiltration

${ }^{d}$ Determined by GPC

\begin{tabular}{lllcrl}
\hline & Feed composition $^{a}$ & Obtained composition $^{b}$ & ${\text { Yield }(\%)^{c}}^{c}$ & $M_{w}(\mathrm{~kg} / \mathrm{mol})^{d}$ & PDI \\
\hline ABOL/EDA & $100 / 0$ & & 27 & 4.1 & 1.4 \\
& $75 / 25$ & $80 / 20$ & 50 & 11.7 & 2.4 \\
& $50 / 50$ & $56 / 44$ & 57 & 7.2 & 1.9 \\
& $25 / 75$ & $30 / 70$ & 53 & 3.1 & 1.6 \\
& & & 45 & 1.6 & 1.6 \\
\hline
\end{tabular}


Fig. 2 Gel retardation assay of siRNA polyplexes at $w / w$ ratio from 0 (siRNA only) to 48, after $\mathrm{I} \mathrm{h}$ incubation in the presence (right) or absence (left) of $5 \mathrm{mM}$ glutathione. Polyplexes were built with polymers containing different percentages of $\mathrm{ABOL} / \mathrm{EDA}$ ( $100 / 0$ (A), 75/25 (B), 50/50 (C), 25/75 (D) and $0 / 100(\mathbf{E}))$.

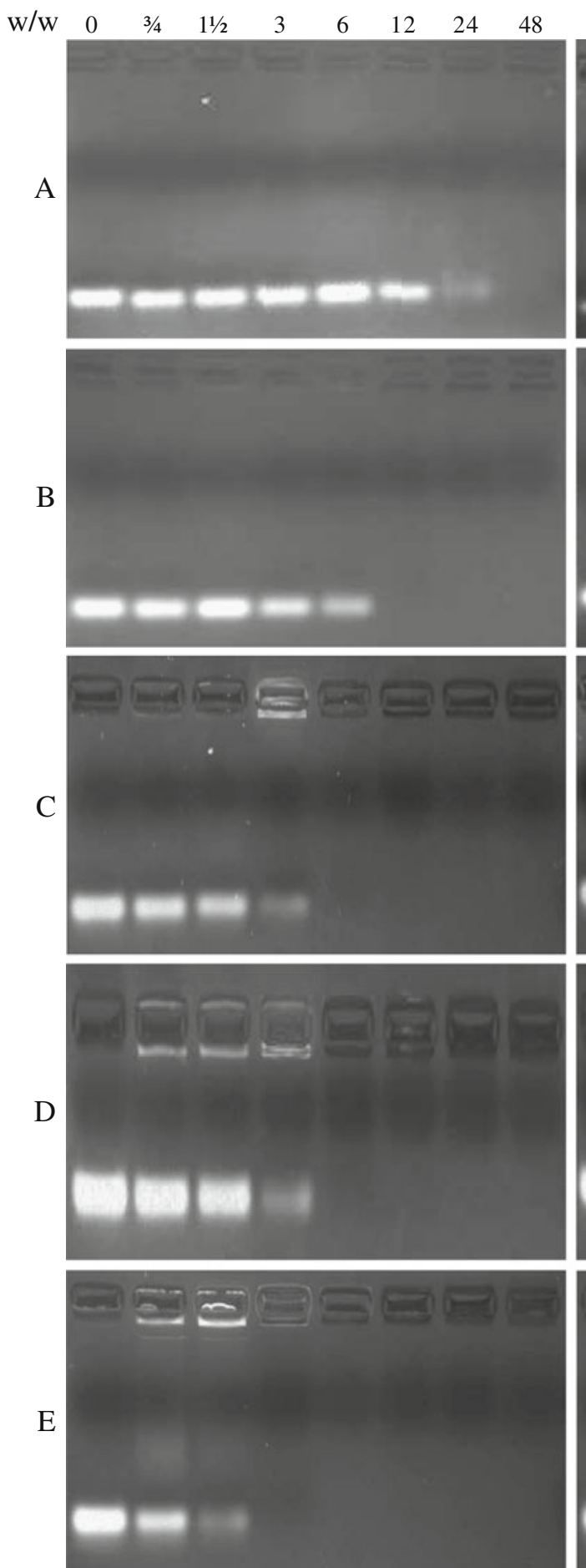

- glutathione
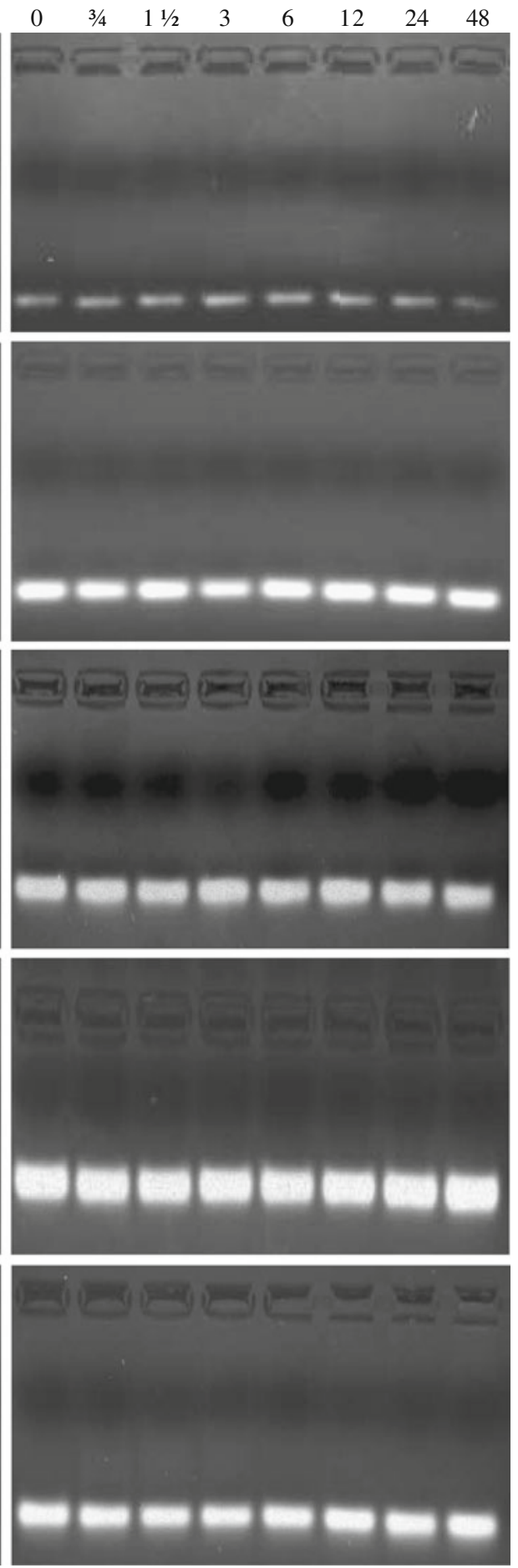

+ glutathione improving the delivery of nucleic acids is the redox potential gradient that exists between the extra- and intracellular environment (20). A high concentration of glutathione $( \pm$ $5 \mathrm{mM}(21)$ ) inside cells causes rapid cleavage of disulfide bonds. The disulfide bridges in the poly(CBA-ABOL/EDA) copolymers are also expected to be cleaved in a reductive environment. To investigate whether this reductive degra- dation also results in siRNA release from the complex, polyplexes were incubated for $1 \mathrm{~h}$ at $37^{\circ} \mathrm{C}$ in the presence of $5 \mathrm{mM}$ glutathione, mimicking the intracellular environment, and subsequently subjected to electrophoresis as described. As shown in Fig. 2, right panel, cleavage of disulfide bridges resulted in release of siRNA for all polymers, even at the highest $w / w$ ratios. These results suggest that siRNA is 
effectively condensated (Fig. 2, left panel), but is released from the complex after successful cellular uptake.

Polyplexes were further characterized by size and $\zeta$ potential measurements. Polyplexes were formed at w/w 3, 6, 12, 24 and 48. Size and surface charge measurements are presented in Fig. 3A and B. For all polymers, increasing the $\mathrm{w} / \mathrm{w}$ ratio resulted in smaller particles with higher $\zeta$ potential. Poly(CBA-ABOL) was only able to condense siRNA into small particles at the higher $\mathrm{w} / \mathrm{w}$ ratios 24 and 48. Polymers with $25 \%$ or $50 \%$ EDA instead of ABOL more efficiently condensed siRNA, resulting in particles smaller than $200 \mathrm{~nm}$ for $\mathrm{w} / \mathrm{w}$ ratio 6 and higher. For the copolymers containing $75 \%$ or $100 \%$ EDA, all polyplexes formed were smaller than $150 \mathrm{~nm}$. Surface charge measurements followed the same trend as the size measurements. For all w/w ratios, $\zeta$-potential of the complexes increased with the amount of EDA in the polymer. For copolymers containing $75 \%$ or $100 \% \mathrm{EDA}$, the $\zeta$-potential of the complexes were all almost equally positively charged
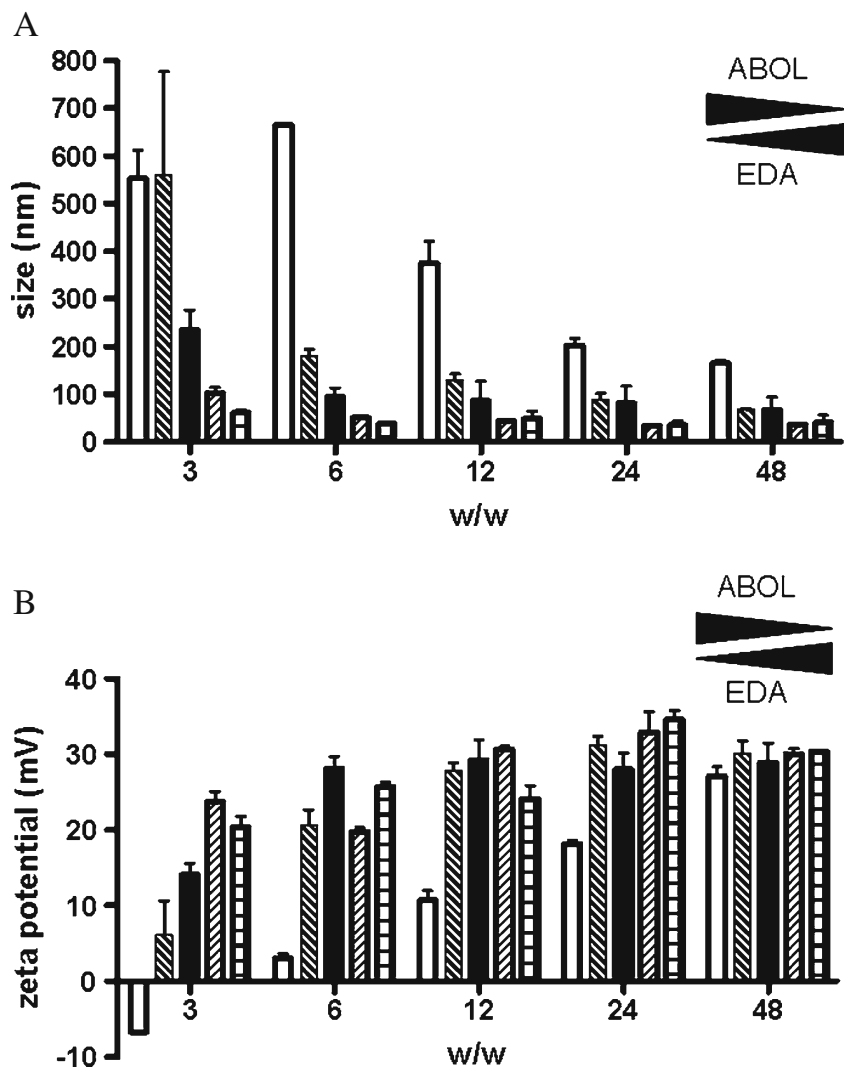

Fig. 3 Particle size $(\mathbf{A})$ and $\zeta$-potential (B) of poly(CBA-ABOL/EDA)/ siRNA polyplexes prepared in $20 \mathrm{mM}$ Hepes $+5 \%$ glucose, at $\mathrm{w} / \mathrm{w}$ ratio of $3,6,12,24$ and 48 . Polyplexes were built with polymers containing different percentages of ABOL/EDA (I00/0 (white bars), 75/25 (black, hatched bars), 50/50 (black bars), 25/75 (white, hatched bars) and 0/I00 (white, blocked bars)). Particle size and $\zeta$-potential were determined by dynamic light scattering and laser Doppler electrophoresis. Results are reported as mean \pm SD for 2-3 individual measurements. to polyplexes at $\mathrm{w} / \mathrm{w}$ ratio 3 , with $\zeta$-potentials between 20 and $30 \mathrm{mV}$. Interestingly, for polymers with $50 \%, 75 \%$ and $100 \%$ EDA, from $\mathrm{w} / \mathrm{w}$ ratio 12,3 and 3 respectively, addition of more polymer to the final complex did not result in smaller particles or higher surface charges. This suggests that from these $\mathrm{w} / \mathrm{w}$ ratios an excess of 'free' polymer was added, which had no effect on the physicochemical properties of the final complex.

\section{Cellular Uptake}

Cellular uptake is one of the key steps in the process of siRNA delivery. To assess the ability of the various polymers to facilitate siRNA internalization, fluorescently labeled siRNA was used to form polyplexes. First, uptake was studied using fluorescent microscopy. The medium was replaced with serum-free medium during the time of transfection $(4 \mathrm{~h})$, as binding of serum proteins to the polyplexes makes it more difficult to exclusively determine the effect of polymer composition on its activity. The fluorescent signal in the cells treated with poly(CBA-ABOL) complexes was very weak. Only at the highest w/w ratios, few cells displayed a faint green staining (Fig. 4A). This indicates that efficient siRNA condensation is a prerequisite for cellular uptake. Introduction of $25 \% \mathrm{EDA}$ in the final polymer resulted in increased uptake of polyplexes at $\mathrm{w} / \mathrm{w}$ ratio 12,24 and 48 . Cells displayed a mixture of discrete green spots and a weaker diffuse green signal, interpreted as siRNA associated with or released from the carrier, respectively (19). At the lowest w/w ratios, fluorescent intensity was still very low (Fig. 4B). Cells transfected with polyplexes formed with polymers with $50 \%$ or $75 \%$ EDA instead of ABOL showed uptake for all w/w ratios; however, at the highest $\mathrm{w} / \mathrm{w}$ ratios, fluorescence was less intense (Fig. 4C, D). For poly(CBA-EDA), fluorescence could only be detected in cells treated with polyplexes at the lowest w/w ratios (Fig. 4E). After uptake of Lipofectamine2000 complexes, used as a control, cells displayed a similar fluorescent signal (Fig. 4F). For all polymers that showed uptake, a diffuse fluorescent signal also appeared, suggesting effective siRNA release from the complexes. Improved cytosolic availability of siRNA may increase the chance of effective gene silencing (22).

Although microscopy images suggested efficient release from the carrier after uptake and showed major differences in uptake efficiency, the ability of different polymers to enhance siRNA internalization was also quantitatively determined. For absolute quantification, fluorescence was measured in cell lysates, since fluorescent signals located in endosomes often cannot be detected due to quenching effects. Furthermore, since complexation of siRNA with polymer also results in loss of fluorescent signal, $2 \%$ sodium dodecyl sulfate (SDS) was added to the lysis buffer, resulting 


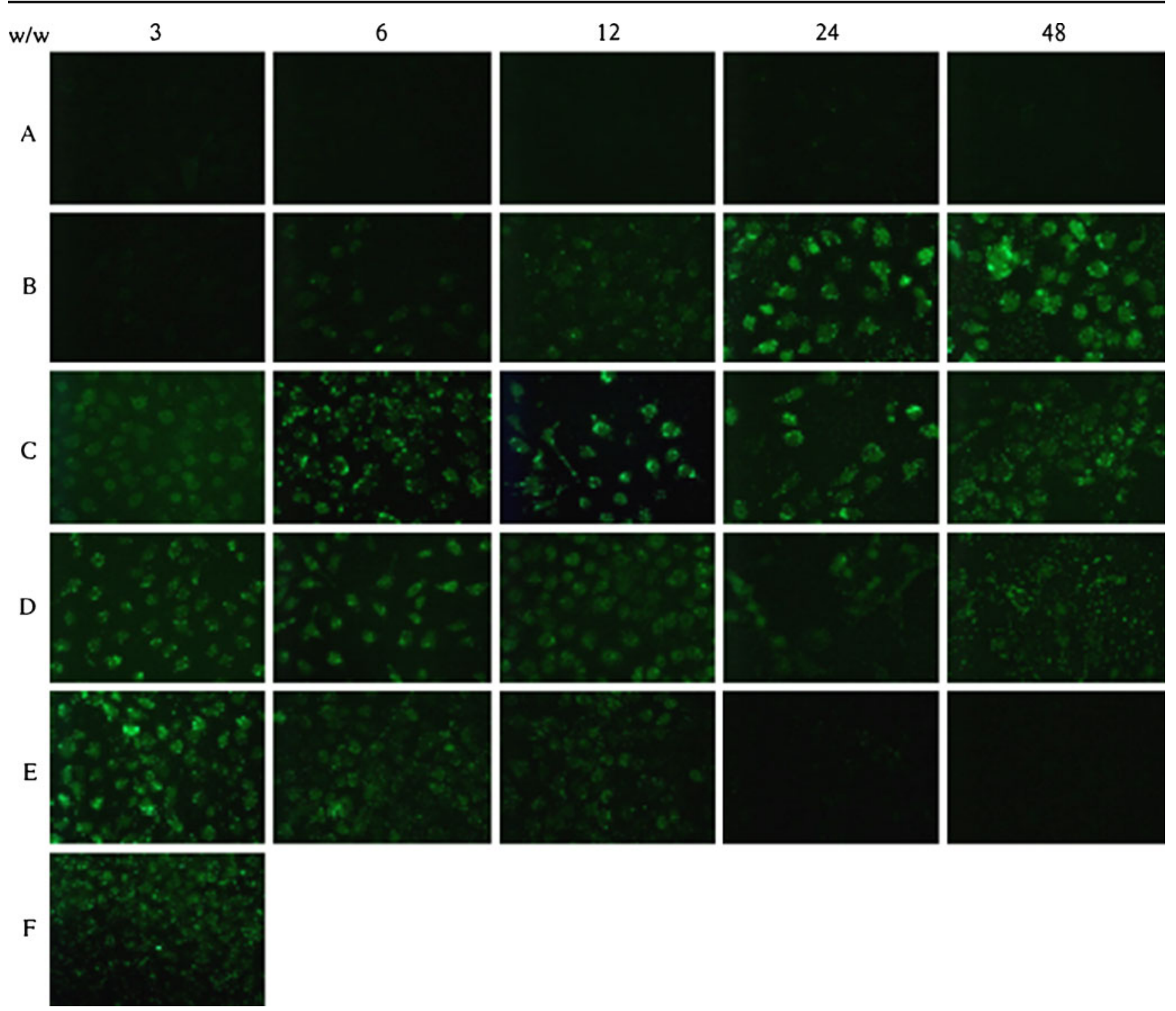

Fig. 4 Fluorescent microscopy pictures of I $4 \mathrm{C}$ cells after treatment with polyplexes at w/w ratio of 3, 6, 12, 24 and 48 . Polyplexes were built with polymers containing different percentages of ABOL/EDA (I00/0 (A), 75/25 (B), 50/50 (C), 25/75 (D) and 0/I 00 (E)). Lipofectamine2000 (F) was used as a control.

in polyplex dissociation and total signal restoration (data not shown). Results are shown in Fig. 5. Consistent with the fluorescent microscopy data, poly(CBA-ABOL) polyplexes showed very little uptake, even at w/w ratio $48(0.3 \%$ of input). Higher percentages of EDA in the polymer resulted in much more effective uptake. For polyplexes formed with the copolymer containing 75\% ABOL and 25\% EDA, uptake increased with increasing $\mathrm{w} / \mathrm{w}$ ratio to a maximum of $14 \%$. Surprisingly, for polymers containing $50 \%, 75 \%$ or $100 \%$ EDA, polyplexes were most efficiently taken up at lower $\mathrm{w} / \mathrm{w}$ ratios, with uptake maxima of $15 \%, 10 \%$ and $7 \%$ at w/w 12, 3 and 3, respectively. As mentioned above, for these polymers complete condensation of siRNA was established at these $\mathrm{w} / \mathrm{w}$ ratios. Thus, at higher $\mathrm{w} / \mathrm{w}$ ratios, free polymer may have hampered siRNA uptake, and this effect is expected to be pronounced in the case of polymers with high charge densities, as was also found in the fluorescent microscopy pictures (Fig. 4C, D and E). This is most likely due to shielding of the heparin surface proteoglycans present on cells by free polymer, resulting in reduced polyplex association, as has been reported earlier for PEI-polyplexes (23). Alternatively, as colloidal stability of the polyplexes increases with increasing $\mathrm{w} / \mathrm{w}$ ratio, decreased aggregation of the polyplexes could result in lower uptake. However, such decreased aggregation at higher w/w ratio was not observed under the conditions used in our transfection studies, as is shown for polyplexes prepared with polymers containing $\mathrm{ABOL} / \mathrm{EDA}$ ratios of 
75/25 and 0/100 (Supplementary Fig. 2). In general, uptake maxima were comparable to uptake of Lipofectamine2000 Lipo-plexes (12\%).

\section{Gene Silencing and Toxicity}

Silencing experiments were performed on a $14 \mathrm{C}$ carcinoma cell line, using siRNA against the epidermal growth factor receptor (EGFR) as an example of a therapeutic target. EGFR is overexpressed in many tumors and is known to play a role in cell proliferation, migration, angiogenesis development and inhibition of apoptosis (24). Polyplexes were formed at w/w 3, 6, 12, 24 and 48 for all polymers. As shown in Fig. 6, for all polymers, increasing the w/w ratio resulted in decreased expression of EGFR after transfection. This effect was specifically caused by siRNA against EGFR, as treatment with the same polyplexes containing negative control siRNA had no effect on EGFR expression, as shown for w/w ratio 48 (Fig. 6B). The same amount of polymer without siRNA also did not decrease EGFR expression (Supplementary Fig. 3). In contrast to our data on siRNA uptake (Figs. 4 and 5), for all polymers, maximal silencing efficiency was obtained at the highest $\mathrm{w} / \mathrm{w}$ ratio tested. Therefore, uptake of polyplexes does not seem to be the only limiting step in the transfection process. Another major hurdle for transfection is known to be endosomal escape. These poly(amido amine)s contain amino groups with different $\mathrm{pK}_{\mathrm{a}} \mathrm{s}$, introducing high buffering capacity for membrane disruption, either via the proton sponge effect (25) or conformational changes induced by $\mathrm{pH}$ changes (26). Our results indicate that a certain threshold amount of polymer is necessary to achieve efficient endosomal escape. For example, while for the polymer containing 50\% ABOL

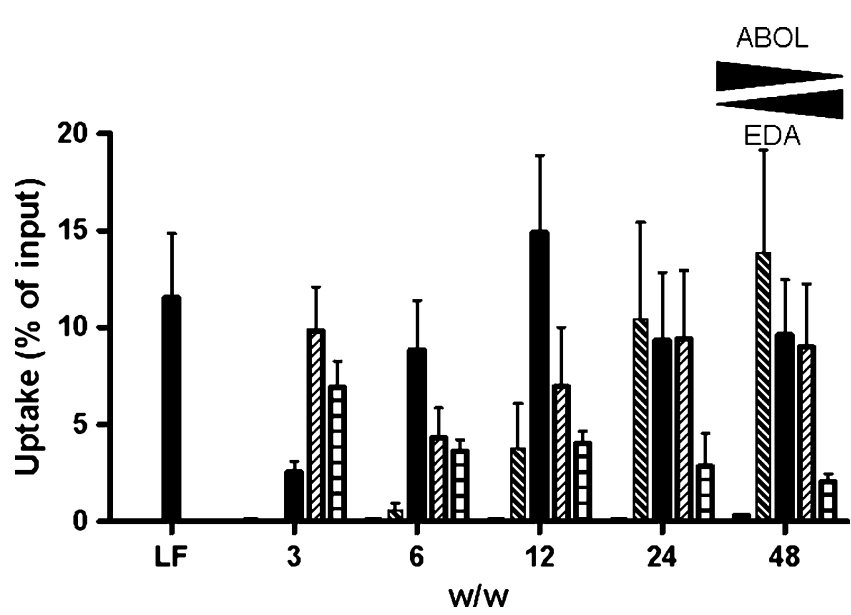

Fig. 5 siRNA uptake in $14 \mathrm{C}$ cells. Cells were treated with polyplexes at w/W ratio of 3, 6, 12, 24 and 48. Polyplexes were built with polymers containing different percentages of ABOL/EDA (100/0 (white bars), 75/25 (black, hatched bars), 50/50 (black bars), 25/75 (white, hatched bars) and 0/100 (white, blocked bars)). Lipofectamine2000 (LF) was used as a control. Uptake was reported as mean uptake \pm SD for $n=3-4$. and 50\% EDA siRNA uptake was higher at $\mathrm{w} / \mathrm{w}$ ratio 12 than at w/w ratio 48, silencing efficiency was much less (approximately 30\% versus $80 \%$, respectively). Likely, the addition of extra polymer contributed to the overall buffer capacity of the complexes inside endosomes. Our data suggest that free polymer, as in the case of polyplexes formed with polymers containing more than 50\% EDA at the highest $\mathrm{w} / \mathrm{w}$ ratios, can also have a positive effect on endosomal escape and subsequent gene silencing. Similar results have been shown for PEI (27).

Interestingly, striking differences in maximum silencing efficiency were found between the different copolymers. Polyplexes formed with polymers containing $25 \%$ or $50 \%$ EDA were able to induce $>80 \%$ EGFR silencing, which was even slightly better than for Lipofectamine2000 lipoplexes, the current standard for in vitro siRNA delivery. Only moderate silencing (approximately 40\%) was found for siRNA formulated with the other polymers. The same trend was observed when cells were transfected in the presence of serum. Only treatment with polyplexes prepared at w/w ratio 48 with polymers containing $25 \%$ or $50 \%$ EDA resulted in EGFR knockdown, although at higher siRNA concentration (200 nM) (Supplementary Fig. 4).

For poly(CBA-ABOL) and poly(CBA-EDA) complexes, only moderate silencing in the absence of serum can be attributed to their poor uptake (Figs. 4 and 5). On the contrary, the amount of siRNA that was taken up after complexation with the polymer containing 75\% EDA was almost equal to the amount taken up after polyplex formation with the polymers with $25 \%$ or $50 \%$ EDA (Fig. 5). Of the three polymers, the first has the highest amount of amine groups and therefore the strongest interaction with siRNA. It is likely that, despite reduction of disulfide bonds in the cytoplasm, the inability to completely release siRNA limited silencing efficiency for this polymer. Likewise, of the three bioreducible polymers that were tested by Christensen et al., the polymer with the highest charge densities of amines produced the lowest overall gene expression (15).

Relative cell viability was evaluated by determining the ability of cells to proliferate after treatment. As show in Fig. 7, polyplexes from polymers with $0 \%$ or $25 \%$ EDA were non-toxic, even at the highest $\mathrm{w} / \mathrm{w}$ ratios. For the polymer with $50 \%$ EDA, polyplexes reduced cell proliferation to approximately $50 \%$ at $\mathrm{w} / \mathrm{w}$ ratio 48 . Polymers with $75 \%$ or $100 \%$ EDA were the most toxic, with respectively $40 \%$ and $10 \%$ viability after treatment with polyplexes at w/w ratio 24 and only a few percent viable cells at w/w 48. In general, polyplexes were more toxic at increasing $\mathrm{w} / \mathrm{w}$ ratio. Furthermore, increasing the amount of EDA in the polymer also resulted in decreased cell viability. Toxicity has previously been shown to be related to charge density of the polymer (28). Comparing these results with the results from the 
A

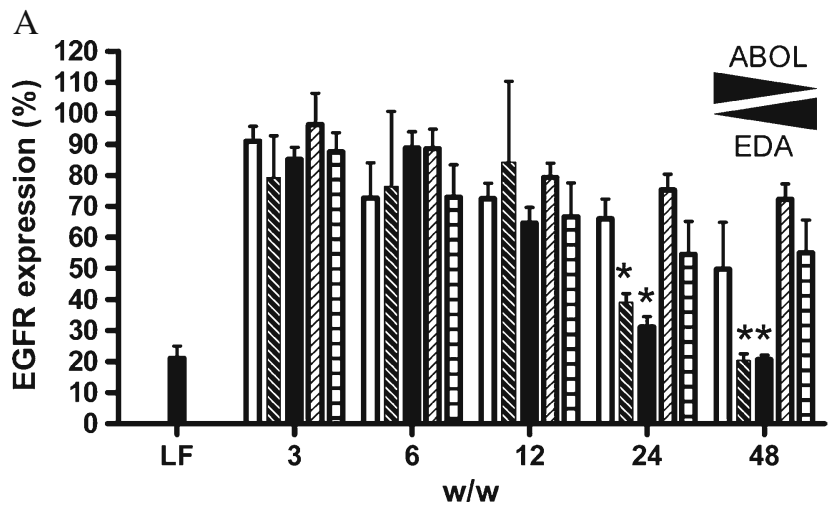

B

B

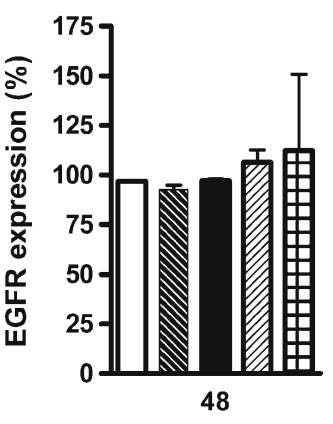

Fig. 6 Gene silencing of EGFR in I4 C cells at a final concentration of $66 \mathrm{nM}$ siRNA. Cells were treated with polyplexes at w/w ratio of 3, 6, I2, 24 and 48 using EGFR siRNA (A) or w/w 48 using negative control siRNA (B). Polyplexes were built with polymers containing different percentages of ABOL/ EDA (I00/0 (white bars), 75/25 (black, hatched bars), 50/50 (black bars), 25/75 (white, hatched bars) and 0/I00 (white, blocked bars)). Lipofectamine2000 (LF) was used as a control. EGFR expression was analyzed by flow cytometry and reported as mean expression \pm SD for $n=2-6$. Statistically significant differences of polymers containing ABOL/EDA percentages of 75/25 and 50/50 from other polymers are denoted by *.

physicochemical characterization of the complexes, toxicity might be primarily caused by addition of an excess of polymer, an observation that is confirmed by other studies (23). Interestingly, while often transfection efficiency seems to be correlated to toxicity, in our study the most toxic polymers were shown to be the least effective.

In the discussion of the results, differences in molecular weight between the polymers were not taken into account, although molecular weight has shown to be of influence on siRNA condensation and toxicity (29). However, the obtained results can be explained by differences in chemical composition between the polymers, as complexation effi-

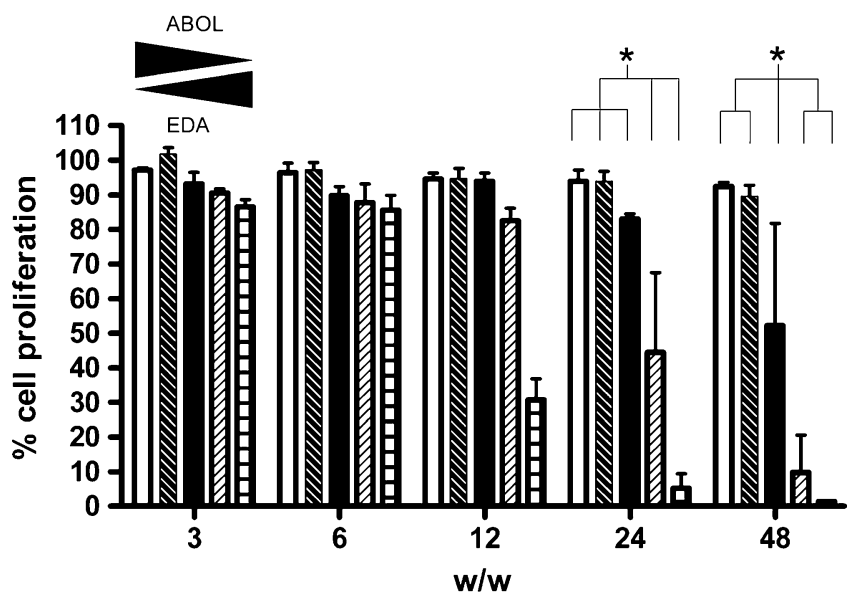

Fig. 7 Percentage of cell proliferation of $14 \mathrm{C}$ cells after treatment with poly(CBA-ABOL/EDA) / siRNA polyplexes, evaluated using the BrdU proliferation assay. Polyplexes were built with polymers containing different percentages of ABOL/EDA (100/0 (white bars), 75/25 (black, hatched bars), 50/50 (black bars), 25/75 (white, hatched bars) and 0/100 (blocked bars)). Results are reported as mean $\pm S D$ for $n=3$. Statistically significant differences are denoted by * $(p<0.05)$. ciency and toxicity correlate with composition of the polymers, but not with molecular weight.

\section{CONCLUSIONS}

In this study, several bioreducible poly(amido amine) copolymers were tested for their ability to deliver siRNA to a carcinoma cell line and induce gene silencing. These copolymers were based on a previously described polymer poly(CBA-ABOL), which has shown to be able to efficiently transfect pDNA. Copolymers with different percentages of EDA and ABOL were synthesized, and the interaction with relatively small siRNA molecules was studied. As predicted, poly(CBA-ABOL) was not able to completely condense siRNA into nano-sized polyplexes, resulting in minor cellular uptake and gene silencing. Addition of $25 \%$ or $50 \% \mathrm{EDA}$ instead of ABOL to the polymer resulted in polyplexes from respective $\mathrm{w} / \mathrm{w}$ ratios of 12 and higher and 6 and higher. These polyplexes were efficiently taken up by $14 \mathrm{C}$ cells. Transfection resulted in effective gene silencing at higher $\mathrm{w} / \mathrm{w}$ ratios with only minor toxicity. Polymers with high percentages of $\mathrm{EDA}(75 \%$ or $100 \%$ ) formed small polyplexes from the lowest $\mathrm{w} / \mathrm{w}$ ratios. Efficient uptake was only found at low $\mathrm{w} / \mathrm{w}$ ratios, indicating that an excess of polymer hindered uptake at higher w/w ratios. However, uptake of polyplexes with low w/w ratios did not result in effective gene silencing, probably due to lack of sufficient polymer to induce endosomal escape.

Taken together, we have shown that introduction of extra amine groups in the CBA-ABOL polymer increased siRNA condensation, resulting in complete shielding and smaller, more positively charged polyplexes at lower $\mathrm{w} / \mathrm{w}$ ratios. Only siRNA that was effectively complexed was taken up by 
cells; however, excess of polymer hindered this process. At the same time, sufficient amount of polymer was necessary for endosomal escape. Our data indicate that CBA-ABOL polymers with a minor amount of EDA are most feasible for siRNA delivery. In the future, the potential of these polymers for their use for in vivo siRNA delivery will be evaluated.

\section{ACKNOWLEDGMENTS}

This project is financially supported by the Technology Foundation STW of The Netherlands Organization for Scientific Research NWO grant UFA7468.

Open Access This article is distributed under the terms of the Creative Commons Attribution Noncommercial License which permits any noncommercial use, distribution, and reproduction in any medium, provided the original author(s) and source are credited.

\section{REFERENCES}

1. Fire A, Xu S, Montgomery MK, Kostas SA, Driver SE, Mello CC. Potent and specific genetic interference by double-stranded RNA in Caenorhabditis elegans. Nature. 1998;391:806-11.

2. Elbashir SM, Harborth J, Lendeckel W, Yalcin A, Weber K, Tuschl T. Duplexes of 21-nucleotide RNAs mediate RNA interference in cultured mammalian cells. Nature. 2001;411:494-8.

3. Meisterand G, Tuschl T. Mechanisms of gene silencing by double-stranded RNA. Nature. 2004;431:343-9.

4. de Fougerolles A, Vornlocher HP, Maraganore J, Lieberman J. Interfering with disease: a progress report on siRNA-based therapeutics. Nat Rev Drug Discov. 2007;6:443-53.

5. Schiffelers RM, Woodle MC, Scaria P. Pharmaceutical prospects for RNA interference. Pharm Res. 2004;21:1-7.

6. Meadeand BR, Dowdy SF. Exogenous siRNA delivery using peptide transduction domains/cell penetrating peptides. Adv Drug Deliv Rev. 2007;59:134-40.

7. Arnold AS, Tang YL, Qian K, Shen L, Valencia V, Phillips MI, et al. Specific betal-adrenergic receptor silencing with small interfering RNA lowers high blood pressure and improves cardiac function in myocardial ischemia. J Hypertens. 2007;25:197-205.

8. Zimmermann TS, Lee AC, Akinc A, Bramlage B, Bumcrot D, Fedoruk MN, et al. RNAi-mediated gene silencing in non-human primates. Nature. 2006;441:111-4.

9. Schiffelers RM, Ansari A, Xu J, Zhou Q, Tang Q, Storm G, et al. Cancer siRNA therapy by tumor selective delivery with ligandtargeted sterically stabilized nanoparticle. Nucleic Acids Res. 2004;32:e149.

10. Sato A, Choi SW, Hirai M, Yamayoshi A, Moriyama R, Yamano $\mathrm{T}$, et al. Polymer brush-stabilized polyplex for a siRNA carrier with long circulatory half-life. J Control Release. (2007).

11. Lin C, Zhong Z, Lok MC, Jiang X, Hennink WE, Feijen J, et al. Linear poly(amido amine)s with secondary and tertiary amino groups and variable amounts of disulfide linkages: synthesis and in vitro gene transfer properties. J Control Release. 2006;116:130-7.
12. Lin C, Zhong Z, Lok MC, Jiang X, Hennink WE, Feijen J, et al. Novel bioreducible poly(amido amine)s for highly efficient gene delivery. Bioconjug Chem. 2007;18:138-45.

13. Anderson DG, Lynn DM, Langer R. Semi-automated synthesis and screening of a large library of degradable cationic polymers for gene delivery. Angew Chem Int Ed Engl. 2003;42:3153-8.

14. Gary DJ, Puri N, Won YY. Polymer-based siRNA delivery: perspectives on the fundamental and phenomenological distinctions from polymer-based DNA delivery. J Control Release. 2007;121:64-73.

15. Christensen LV, Chang CW, Kim WJ, Kim SW, Zhong Z, Lin C, et al. Reducible poly(amido ethylenimine)s designed for triggered intracellular gene delivery. Bioconjug Chem. 2006;17:1233-40.

16. Vader P, van der Aa LJ, Engbersen JF, Storm G, Schiffelers RM. A method for quantifying cellular uptake of fluorescently labeled siRNA. J Control Release. 2010;148:106-9.

17. Oliveira S, Fretz MM, Hogset A, Storm G, Schiffelers RM. Photochemical internalization enhances silencing of epidermal growth factor receptor through improved endosomal escape of siRNA. Biochim Biophys Acta. 2007;1768:1211-7.

18. Piest M, Lin C, Mateos-Timoneda MA, Lok MC, Hennink WE, Feijen J, et al. Novel poly(amido amine)s with bioreducible disulfide linkages in their diamino-units: structure effects and in vitro gene transfer properties. J Control Release. 2008;130:38-45.

19. Breunig M, Hozsa C, Lungwitz U, Watanabe K, Umeda I, Kato $\mathrm{H}$, et al. Mechanistic investigation of poly(ethylene imine)-based siRNA delivery: disulfide bonds boost intracellular release of the cargo. J Control Release. 2008;130:57-63.

20. Soundara Manickamand D, Oupicky D. Polyplex gene delivery modulated by redox potential gradients. J Drug Target. 2006;14:519-26.

21. Hwang C, Lodish HF, Sinskey AJ. Measurement of glutathione redox state in cytosol and secretory pathway of cultured cells. Methods Enzymol. 1995;251:212-21.

22. Hoon Jeong J, Christensen LV, Yockman JW, Zhong Z, Engbersen JF, Jong Kim W, et al. Reducible poly(amido ethylenimine) directed to enhance RNA interference. Biomaterials. 2007;28:1912-7.

23. Boeckle S, von Gersdorff $\mathrm{K}$, van der Piepen S, Culmsee C, Wagner E, Ogris M. Purification of polyethylenimine polyplexes highlights the role of free polycations in gene transfer. J Gene Med. 2004;6:1102-11.

24. Oliveira S, van Bergen en Henegouwen PM, Storm G, Schiffelers RM. Molecular biology of epidermal growth factor receptor inhibition for cancer therapy. Expert Opin Biol Ther. 2006;6:605-17.

25. Boussif O, Lezoualc'h F, Zanta MA, Mergny MD, Scherman D, Demeneix B, et al. A versatile vector for gene and oligonucleotide transfer into cells in culture and in vivo: polyethylenimine. Proc Natl Acad Sci U S A. 1995;92:7297-301.

26. Griffiths PC, Paul A, Khayat Z, Wan KW, King SM, Grillo I, et al. Understanding the mechanism of action of poly(amidoamine)s as endosomolytic polymers: correlation of physicochemical and biological properties. Biomacromolecules. 2004;5:1422-7.

27. Sonawane ND, Szoka Jr FC, Verkman AS. Chloride accumulation and swelling in endosomes enhances DNA transfer by polyamine-DNA polyplexes. J Biol Chem. 2003;278:44826-31.

28. Fischer D, Li Y, Ahlemeyer B, Krieglstein J, Kissel T. In vitro cytotoxicity testing of polycations: influence of polymer structure on cell viability and hemolysis. Biomaterials. 2003;24:1121-31.

29. Grayson AC, Doody AM, Putnam D. Biophysical and structural characterization of polyethylenimine-mediated siRNA delivery in vitro. Pharm Res. 2006;23:1868-76. 